\title{
Differential metabolome landscape of Kadsura coccinea fruit tissues and potential valorization of the peel and seed tissues
}

\author{
Jianfei $\mathrm{GAO}^{1}$; Kangning XIONG ${ }^{2, *}$; Weijie $\mathrm{LI}^{1}$; Wei $\mathrm{ZHOU}^{3}$ \\ ${ }^{1}$ Institute of Mountain Resources, Guizhou Academy of Sciences, Guiyang, 550001, China \\ ${ }^{2}$ School of Karst Science, Guizhou Normal University, State Engineering Technology Institute for Karst Desertfication Control of China, Guiyang, 550001, China \\ ${ }^{3}$ Guizhou Industry Polytechnic College, Guiyang, 550008, China
}

Key words: Kadsura coccinea, Widely-targeted metabolomics, Alkaloids, Lignans, Flavonoids, Fruit, Biowaste valorization

\begin{abstract}
Kadsura coccinea (Lem.) is a woody wine plant with a peculiar fruit enriched in important health-promoting compounds. The non-editable part of the fruit, i.e., the seed and peel, represents more than $60 \%$ of the fruit and is considered a biowaste. This significantly restricts the development of the $K$. coccinea fruit industry. Clarifying the metabolic components of the different fruit parts can help to improve the utilization rate and valorization of K. coccinea. Herein, we evaluated $K$. coccinea fruit peel, pulp, and seed using widely-targeted metabolomics and quantified a set of 736 bioactive compounds from 11 major metabolite classes. The most prominent metabolite classes included lipids, amino acids, flavonoids, and lignans. Furthermore, our results emphasized a significant accumulation of flavonoids in pulp tissues, while alkaloids and lignans were abundant in peel and seed tissues, respectively. A total of 183 metabolites were differentially accumulated among the three tissues. Procyanidin C2, rutinoside, 2-hydroxyoleanolic acid, 5-hydroxymethyluracil, nootkatol, isoquercitrin, isohyperoside, quercetin-7-O-glucoside, hyperin, and rutin showed elevated accumulation in the peel. In the seed, kadsuralignan G, kadcoccilactone A, kadsuralignan H, lysoPE 20:5, iso-schisandrin ethyl alcohol, and kadangustin were significantly enriched. Our results highlight the diverse metabolome composition of $K$. coccinea fruit parts, which can be further exploited for its valorization in various industries.
\end{abstract}

\section{Introduction}

Black tiger (Kadsura coccinea (Lem.)) is a perennial evergreen climbing woody vine indigenous to Southwest China (Sun et al., 2009) and widely distributed in Guizhou, Guangxi, Yunnan, Guangdong, and Sichuan (Sritalahareuthai et al., 2020a). The mature black tiger fruit is usually consumed as fresh fruit. However, the edible portion of the fruit is only $30-40 \%$, which is relatively lower compared to popular fruits. The high proportion of skin and seed weights of black tiger fruit is one of the main reasons for its low commercial value. Bioactive compounds present in different plant parts are an excellent source for potential valorization and value addition of the fruit plants. Previous studies emphasized valorization of plant biowaste by utilizing a broad spectrum of bioactive compounds present in plant parts generally considered biowaste. Deng et al. (2020) and Wang et al. (2020a), in their studies on banana pseudo-stem

*Address correspondence to: Kangning Xiong, xiongkn@163.com Received: 22 February 2021; Accepted: 02 April 2021 and longan fruit parts (skin and seed), respectively, demonstrated the potential valorization of biowaste by exploiting the metabolome and identified several compounds with nutraceutical properties. Hence, investigating the metabolome composition of black tiger fruit parts could open a new avenue towards a better valorization of the fruit and the development of the industry.

Kadsura genus, with extensive uses in traditional medicine (Yang et al., 2020), is known as an abundant source of lignans and terpenoids (Yang et al., 2019b), exhibiting several biological functions with beneficial properties, i.e., anti-tumor (Liu et al., 2014), cytotoxic (Hu et al., 2016), anti-HIV (Gao et al., 2008b), antioxidative (Cao et al., 2019), anti-hepatitis (Kuo et al., 2005) and hepatoprotective effects (Liu et al., 2018a). K. coccinea is an important raw material for traditional Chinese medicine. Different plant parts of $K$. coccinea, including rhizomes, bark, stem, and fruit, have been widely used as raw ingredients for the formulation of traditional Chinese medicine (Li et al., 2006; Li et al., 2007; Yang et al., 2020) and subsequently utilized for the treatment of ulcer, acute gastroenteritis, rheumatoid arthritis, bruise, pain, and 
dysmenorrhea (Wang et al., 2020b). However, there is a lack of studies concerning the different parts of the fruit and their potential utilization as dietary or medicinal sources.

The fruit of the black tiger, with its peculiar shape, appearance, bright color, and unique flavor, possesses high health and medicinal values (Hao et al., 2014; Sritalahareuthai et al., 2020a; Sun et al., 2009). Black tiger fruit is highly nutritive, with a higher vitamin $\mathrm{C}$ content as compared to common fruits such as apple, cherry, grape, Kiwi, litchi, and strawberry (El-Ishaq and Obirinakem, 2015). In our preliminary study, we found some biological activities of black tiger fruit peel and seed extracts. If the metabolic components of black tiger fruit parts are elucidated, the value of the nonedible part can be further explored with potential utilization in the food and pharmaceutical industries. Therefore, this study was designed to aim at exploring the differential metabolome of fruit parts, including peel, pulp, and the seed of black tiger fruit, based on the widely-targeted metabolomics approach.

\section{Materials and Methods}

\section{Plant material}

The black tiger plants, locally known as 'HeiLaohu', used in the experiment, resulted from 6 years of artificial cultivation. They were planted in an $80 \%$ plastic canopy with a sunshade net. The experiment was conducted in Xiaba, Wudang District, Guiyang, China, with regular management practices. Randomly selected fruits were picked during the maturation season in October 2020. Each selected fruit was rinsed with tap water, placed on a small plate, and cut with a scalpel. The peel and the pulp parts were slowly stripped out and collected separately into $10 \mathrm{~mL}$ centrifuge tubes. The seeds were rubbed in an emery cloth for seed tissue sampling to remove the flesh and then put into a $10 \mathrm{~mL}$ centrifuge tube. Three biological repeats for each sample from the peel, pulp, and seed were collected from three different fruits. Samples were stored at $-80^{\circ} \mathrm{C}$ for further use.

\section{Sample preparation}

The samples were prepared according to Deng et al. (2020). The three groups of samples, cryopreserved, were freeze-dried in a vacuum using lyophilizer (Scientz-100F) and ground to powder. A $100 \mathrm{mg}$ powder was accurately weighed and thawed in $1.2 \mathrm{~mL} \mathrm{70 \%} \mathrm{methanol} \mathrm{extract} \mathrm{(Shanghai} \mathrm{Aladdin}$ Bio-Chem Technology Co., Ltd., Shanghai, China). The samples were centrifuged every $30 \mathrm{~min}$ for $30 \mathrm{~s}$, a total of six times. The samples were placed in a refrigerator at $4^{\circ} \mathrm{C}$ overnight. After centrifugation, at $12000 \mathrm{rpm}$ for $10 \mathrm{~min}$, the supernatant was absorbed, and later, samples were filtered with $0.22 \mu \mathrm{m}$-pore size (ALWSCI Technologies Zhejiang, China) and stored in injection bottles for further analysis.

\section{UPLC-MS/MS analysis}

The ultraperformance liquid chromatography-tandem mass spectrometry (UPLC-MS/MS performed by Metware, Wuhan, China) was used as a widely targeted metabolomics approach to detect and quantify the metabolome in the three black tiger fruit tissues including, peel, pulp, and seed. The sample extracts were analyzed using a UPLC-ESI-MS/MS system (UPLC, SHIMADZU NexeraX2, www.shimadzu.com.cn/; MS,
Applied Biosystems 4500 Q TRAP, www.appliedbiosystems. com.cn/). The analytical conditions were as follows, UPLC: column, Agilent SB-C18 $(1.8 \mu \mathrm{m}, 2.1 \mathrm{~mm} \times 100 \mathrm{~mm})$; The mobile phase consisted of solvent A and solvent B. Solvent A consisted of pure water with formic acid $(0.1 \%)$, and solvent B consisted acetonitrile with formic acid (0.1\%) (Shanghai Aladdin Bio-Chem Technology Co., Ltd., Shanghai, China). The gradient program was used to measure the samples with a starting point of $95 \% \mathrm{~A}, 5 \% \mathrm{~B}$. After $9 \mathrm{~min}$, the linear gradient was changed to $5 \% \mathrm{~A}, 95 \% \mathrm{~B}$ for $1 \mathrm{~min}$. Later, at $1.10 \mathrm{~min}$, the gradient was adjusted back to $95 \%$ A, $5.0 \%$ B for $2.9 \mathrm{~min}$. The flow velocity was kept constant during the whole gradient process as $0.35 \mathrm{~mL} / \mathrm{min}$. Moreover, the temperature was also kept constant at $40^{\circ} \mathrm{C}$, while the injection volume was $4 \mu \mathrm{L}$.

AB4500 Q TRAP UPLC/MS/MS (triple quadrupolelinear ion trap mass spectrometer) system was utilized to perform ESI-triple quadrupole-linear ion trap (ESI-Q TRAP-MS/MS). The system was equipped with an ESI turbo Ion spray interface. Analyst 1.6.3 was used to control the system (Xu et al., 2020).

\section{ESI-Q TRAP-MS/MS}

For ESI-triple quadrupole-linear ion trap (QTRAP)-MS, LIT, and triple quadrupole (QQQ) scans were acquired on a triple quadrupole-linear ion trap mass spectrometer ( $Q$ TRAP), AB4500 Q TRAP UPLC/MS/MS System, equipped with an ESI Turbo Ion-Spray interface, operating in positive and negative ion mode and controlled by Analyst 1.6.3 software (AB Sciex). The ESI source operation parameters were kept as explained by Chen et al. (2013).

\section{Quality control and data analysis}

To confirm the reliability of metabolome data sets, quality control was performed, as previously described by Fiehn et al. (2008). Extracted samples were mixed and inserted into every sample, and changes were monitored (Yang et al., 2019a). Data sets with the intensity of the metabolites from the three samples were uploaded to the Analyst 1.6.3 software ( $A B$ SCIEX, Ontario, ON, Canada) for descriptive statistical analyses (Dossa et al., 2020). Unsupervised principal component analysis (PCA) was performed by statistics function prcomp within $\mathrm{R}$ (www.r-project.org).

\section{Differential metabolites}

Significantly differentially accumulated metabolites between the fruit tissues were estimated by exploring the variable importance in projection (VIP) values greater than 1 with $\log _{2}$ fold change (FC) also greater than 1. Before Orthogonal Projections to Latent Structures-Discriminant Analysis (OPLS-DA), data was transformed as $\log _{2} \mathrm{FC}$, and mean centering was also performed. OPLS-DA results were used for the extraction of VIP values using $\mathrm{R}$ software with MetaboAnalystR package. Subsequently, 200 permutations were performed for each sample.

\section{Results}

Metabolic landscape in black tiger fruit parts

The UPLC-MS/MS technique was employed for metabolic profiling of black tiger fruit parts viz., peel, pulp, and seed 
(Fig. 1). Repeatability of metabolites detection was ensured by observing the accuracy of the instrument using quality control, as previously explained by Fiehn et al. (2008). We observed overlapped peaks of the total ion current in different quality control samples (Fig. 2a). The results depicted overlapped and consistent peaks for ion-intensity peaks (showed on the y-axis) and retention time. Furthermore, the analysis showed stable peaks for identical samples for different time intervals.

Principal component analysis (PCA) was executed to evaluate the structure of the data. The PCA plot showed the distribution of three groups based on metabolite ion intensity in specific tissues (Fig. 2b). We observed a close grouping of biological replicates in each tissue, while all three tissues viz., peel, pulp, and seed were in separate groups, suggesting that high-quality metabolome data was obtained. Metabolome variance explained by the first two PCs was accumulatively high (90.34\%).

After a quality check, we identified 736 metabolites, grouped into 11 major classes and 35 sub-classes (Fig. 3 and Tab. S1). The classification was based on the structural configuration of the identified metabolites. Of the 736 metabolites, lipids (17.66\%) showed the highest proportion, followed by phenolic acids and derivatives (16.17\%), amino acids and derivatives (13.18\%), flavonoids (11.55\%), organic acids $(10.87 \%)$, others $(10.05 \%)$, nucleotides and derivatives (6.52\%), lignans and coumarins (5.57\%), terpenoids (4.35\%), alkaloids (2.17\%), and tannins (1.90\%) (Fig. 3). Complete information about the set of identified metabolites, including molecular weights $(\mathrm{Da})$, compound formula, ionization, compounds, classes, and KEGG

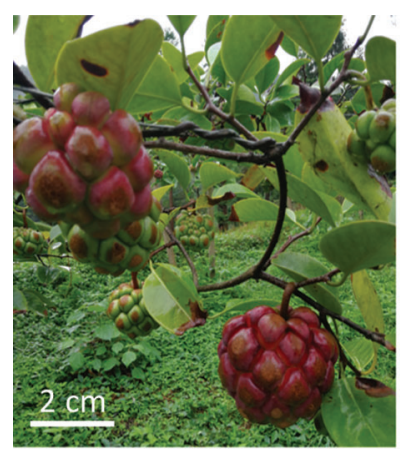

(a)

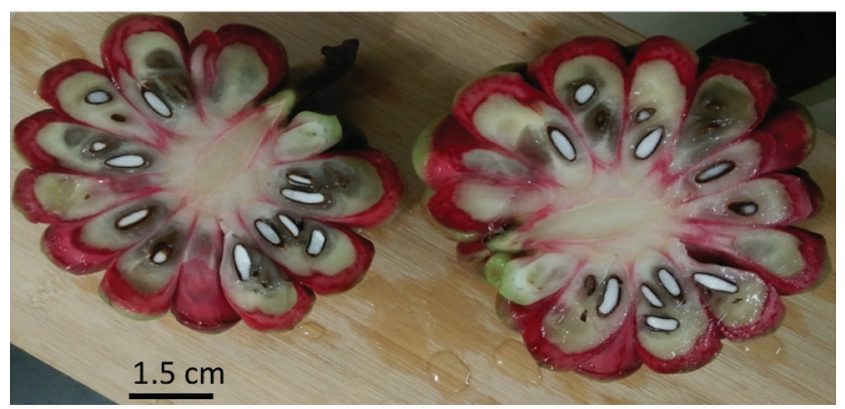

(c)

FIGURE 1. A pictorial description of black tiger fruit (Kadsura coccinea (Lem.)).

(a-b) Black tiger fruit. (c) Cross section of black tiger fruit. pathways, are listed in Tab. S1. The concentration (ion intensity) of the identified metabolites has been presented as a heatmap (Fig. 4), depicting their differential accumulation across the three tissues. Flavonoids, phenolic acids, terpenoids, and tannins showed relatively higher concentrations in the peel than in the pulp and seed. However, lignans and coumarins showed the highest accumulation in the seed.

\section{Differential metabolic landscape}

In order to understand the metabolic properties of the three fruit tissues, we identified the differentially accumulated metabolites (DAMs) between the tissues. In KF vs. KP, we obtained 299 DAMs (Tab. S2), 492 DAMs in KF vs. KD (Tab. S3), and 533 DAMs in KP vs. KD (Tab. S4). We further identified 183 conserved DAMs between the three groups (Fig. 5 and Tab. S5). These conserved DAMs can be categorized into ten major metabolites classes viz., alkaloids, amino acids, flavonoids, lignans and coumarins, lipids, nucleotides and derivatives, organic acids, phenolic acids, tannins, and terpenoids. The differential accumulation profile of these conserved metabolites has been illustrated in Fig. S2, indicating an up-accumulation of 114 DAMs in the peel (Tab. S5). While 37 and 38 DAMs were upaccumulated in the seed and pulp, respectively. This indicates that the peel contains more bioactive compounds even than the pulp. The most noticeable DAMs between pulp and peel were procyanidin $\mathrm{C} 2$, quercetin-3-O-(2")-Oarabinosyl) rutinoside, 2-hydroxyoleanolic acid, 5hydroxymethyluracil, nootkatol, quercetin-3-O-glucoside (isoquercitrin), isohyperoside, quercetin-7-O-glucoside, quercetin-3-O-galactoside (hyperin), and quercetin-3-Orutinoside (rutin).

Further, we explored the metabolome of black tiger fruit by comparing differential accumulation between pairs of tissues. Comparison of pulp and peel (KF vs. KP) yielded 299 DAMs (Tab. S2). The accumulation profile of these DAMs has been presented as a heatmap (Fig. 6a) with corresponding classes. A total of 168 DAMs were found with an up-accumulation pattern in the peel, while 131 DAMs were up-accumulated in the pulp. The top 10 DAMs up-accumulated in peel were flavonoids: delphinidin-3-Oglucoside (mirtillin), isorhamnetin-3-O-glucoside, myricetin3-O-glucoside, quercetin-3-O-(2"-O-rhamnosyl) rutinoside, quercetin-3-O-rutinoside-7-O-rhamnoside, taxifolin-3'-Oglucoside, delphinidin-3-O-(2"-O-glucosyl) rutinoside, quercetin-3-O-sambubioside, eriodictyol-3'-O-glucoside, and tannins: procyanidin C2 (Fig. 6b). In contrast, the pulp showed a high accumulation of L-alanine, kadangustin I, kadangustin G, eicosadienoic acid, acetylepigomisin $\mathrm{R}$, pregomisin, kadangustin $\mathrm{F}$, kadusurain $\mathrm{C}$, kadsuralignan $\mathrm{J}$, and N-a-acetyl-L-ornithine (Fig. 6b).

Similarly, a comparison of pulp and seed resulted in the identification of 492 DAMs, with 115 metabolites upaccumulated in the seed while 377 were up-accumulated in the pulp (Fig. $7 \mathrm{a}$ and Tab. S3). Kadsuralignan G, kadcoccilactone A, kadsuralignan $\mathrm{H}$, lysoPE 20:5, isoschisandrin ethyl alcohol, kadangustin $\mathrm{H}$, gomisin $\mathrm{G}$, schisandrol B, maslinic acid, and plaunol E showed strong accumulation in the seed (Fig. 7b). While, p-coumaric acid, 


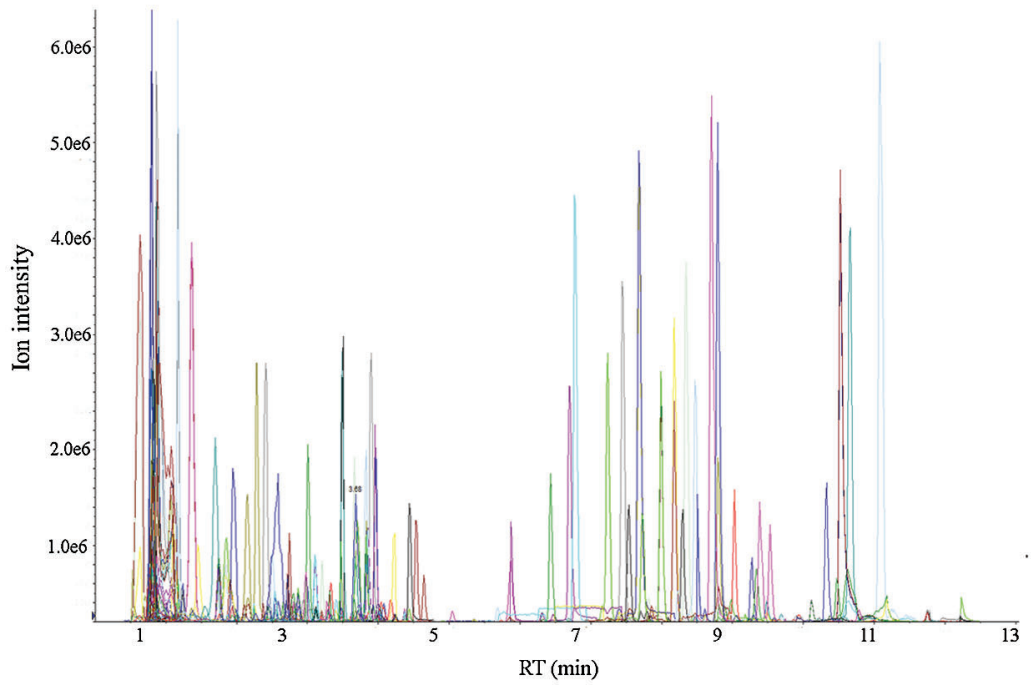

(a)

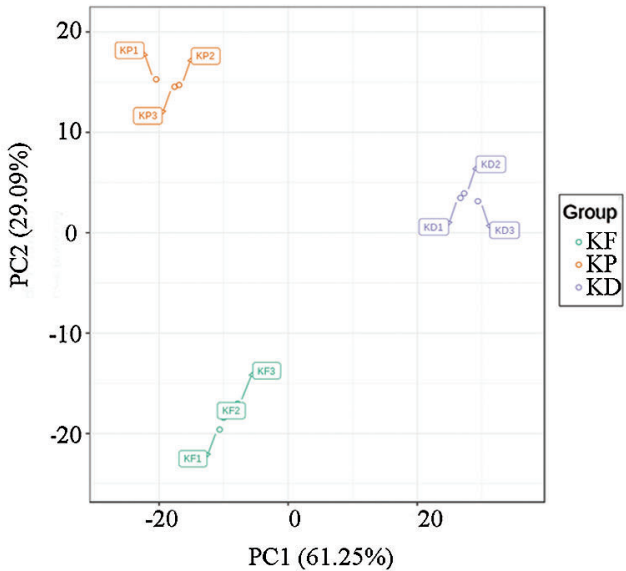

(b)

FIGURE 2. Quality control analysis for consistent metabolites measurements.

(a) The vertical axis represents the intensity of the ion current (cps: count per second), and the horizontal axis shows the retention time (min);

(b) Principal component analysis for metabolites identified in three different tissues of black tiger fruit viz. KP (peel), KF (pulp), and KD (seed).

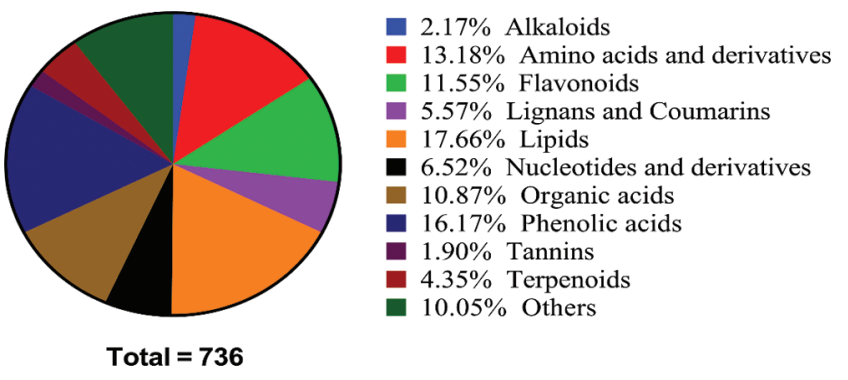

FIGURE 3. Pie chart representing the identified 736 metabolites and the corresponding major classes.

kadcoccinic acid G, delphinidin-3-O-(2"-O-xylosyl)rutinoside, cyanidin-3-O-(2"-O-glucosyl)rutinoside, cyanidin-3-O-rutinoside (keracyanin), kadcotrione C, cyanidin-3-O-glucoside (kuromanin), kadcoccitone C, lariciresinol-4'-O-glucoside, and rosmarinic acid-3'-O-glucoside showed up-accumulation in the pulp as compared to the seed (Fig. 7b).

Comparison of seed and peel (KP vs. KD) metabolome resulted in the identification of 533 DAMs, with 144 showing up-accumulation in the seed while 389 showed upaccumulation in the peel tissues (Tab. S4). The accumulation level of the identified DAMs between peel and seed has been presented in Fig. 8a. Pregomisin, kadangustin G, kadsuralignan $G$, kadangustin $F$, kadusurain $C$, kadsuralignan J, kadcoccilactone A, kadangustin I, kadsuralignan $\mathrm{H}$, and acetylepigomisin $\mathrm{R}$ were among the up-accumulated metabolites in the seed (Fig. 8b). Epicatechin, 4-aminobenzoic acid, cyanidin-3-O-(2"-Oglucosyl) rutinoside, delphinidin-3-O-glucoside (mirtillin), cyanidin-3-O-rutinoside (keracyanin), quercetin-3-Orutinoside (rutin), rosmarinic acid-3'-O-glucoside, lariciresinol-4'-O-glucoside, delphinidin-3-O-(2"-O-xylosyl) rutinoside and cyanidin-3-O-glucoside (kuromanin) showed up-accumulation in the peel compared to the seed (Fig. 8b).

Above mentioned metabolome comparisons suggested that peel tissues of black tiger fruit have more diverse and concentrated metabolites, particularly flavonoids compared to the pulp and seed. On the other hand, the seed also contains elevated amounts of lignans and coumarins.

\section{Discussion}

Metabolites are naturally occurring organic compounds present in living organisms, with the most common being flavonoids, amino acids, alkaloids, and lipids (Erb and Kliebenstein, 2020). In this study, we explored the metabolome of black tiger (Kadsura coccinea (Lem.)) fruit parts viz., peel, pulp, and seed using the widely-targeted metabolomics approach. Widely-targetted metabolomics provides an inclusive platform for identifying and further exploiting diverse metabolomes (Chen et al., 2013; Sawada et al., 2009). The study aimed to provide a comprehensive insight into the differential metabolome of different fruit parts of black tiger fruit, which is used in traditional Chinese medicine due to its antioxidant, anti-tumor, antiHIV anti-lipid peroxidative, cytotoxic, and anti-hepatitis properties (Sritalahareuthai et al., 2020a; Woo et al., 2020).

The metabolome landscape of black tiger fruit revealed an elevated concentration of flavonoids, phenolic acids, terpenoids, and tannins in the peel than pulp and seed. However, lignans and coumarins showed significantly higher accumulation in the seed. Previous reports have also reported the identification of flavonoids (Sritalahareuthai et al., 2020a), phenolic acids (Sun et al., 2009), and lignans (Li et al., 2006) from different tissues of the black tiger plant. Moreover, $K$. coccinea was shown to contain gallic acid (phenolic acid) (Sun et al., 2011), with a strong DPPHradical scavenging activity. The pulp showed a high abundance of flavonoids and lipids, and the most noticeable, with known beneficial effects on human health, are rutinoside (Matsumoto et al., 2001; Olthof et al., 2003), $\gamma$ linolenic acid (Fan and Chapkin, 1998), a-linolenic acid (Burdge, 2006), lysoPE 16:0 (Ren et al., 2018), lysoPC 18:2 


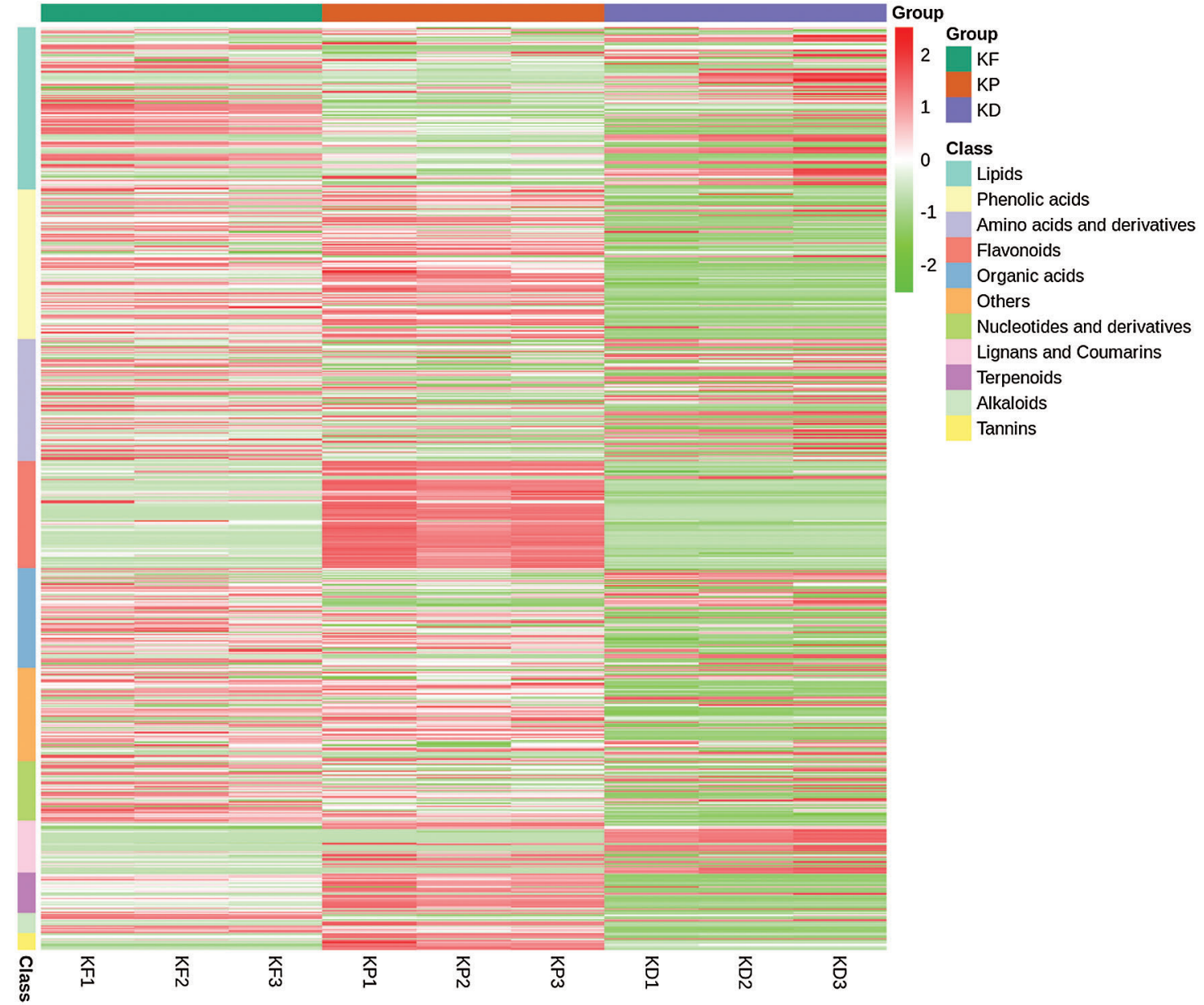

FIGURE 4. Heatmap representing the accumulation (ion intensity) of metabolites in three tissues KP (peel), KF (pulp), and KD (seed).

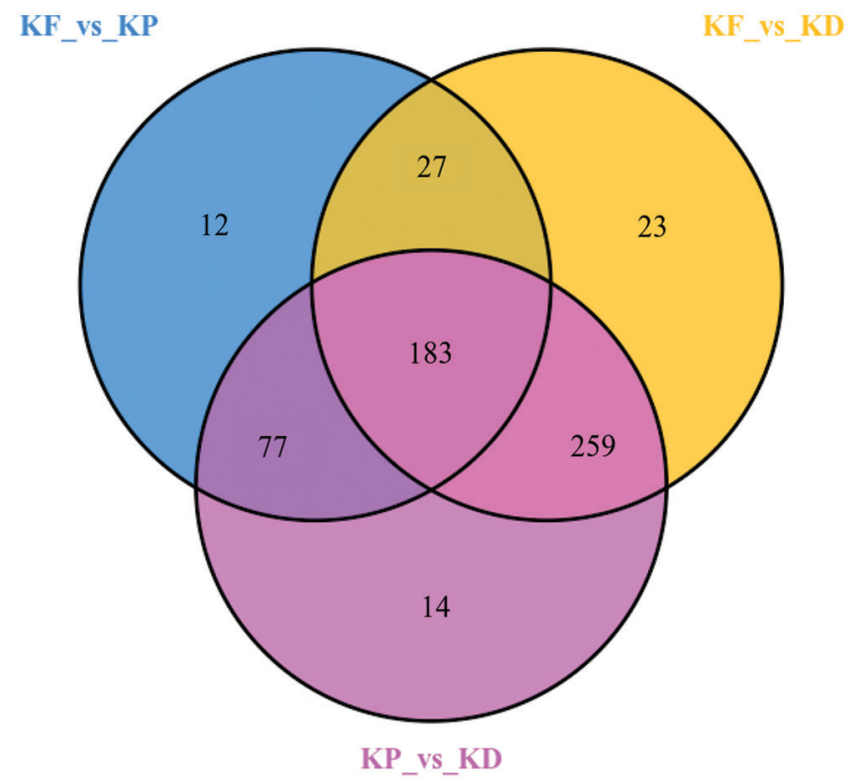

FIGURE 5. Venn diagram representing differentially accumulating metabolites (DAMs) between different tissues of the black tiger: peel (KP), pulp (KF) and seed (KP).

(2n isomer), stearic acid (Bonanome and Grundy, 1988), and lysoPC 18:2 (Dong et al., 2010). Similarly, the peel showed an abundance of flavonoids. Cyanidin-3-O-glucoside, also known as "kuromanin" is responsible for red hue in different fruits (Olivas-Aguirre et al., 2016) and is also known for its antioxidant and anti-tumor characteristics (Sun et al., 2012). Nasser et al. (2019) reported neuroprotective effects of kuromanin chloride (Cyanidin-3O-glucoside) against neurodegenerative diseases. A recent report by Sritalahareuthai et al. (2020b) emphasized the abundance of flavonoids in $K$. coccinea compared to other Kadsura species. Isorhamnetin-3-O-rutinoside (Narcissin) has been recently reported to inhibit COVID-19 virus main protease (Owis et al., 2020). Delphinidin-3-O-(2"-Oxylosyl) rutinoside was also identified in the peel, which is an important flavonoid in the biosynthesis of purple to blue color in plants (Xu et al., 2018). The presence of delphinidin-3-O-(2"-O-xylosyl) rutinoside and cyanidin-3$\mathrm{O}$-glucoside and their higher accumulation in peel tissues are predicted to confer the peculiar red peel color of black tiger fruit. Anthocyanidins are water-soluble pigmented compounds widely distributed in blue to purplish fruits (Sritalahareuthai et al., 2020b), with cyanidin and delphinidin as the most abundant anthocyanidins (Dini et al., 2019). Beyond pigmentation, anthocyanins also play a critical role as therapeutic agents for Central Nervous System (Cásedas et al., 2020). Furthermore, we also identified gallocatechin, which has been previously reported in pomegranate (Plumb et al., 2002), guava (Matsuo et al., 1994), and tea (Ikeda et al., 2003). Gallocatechin is an important flavonoid and displays a 
(a)

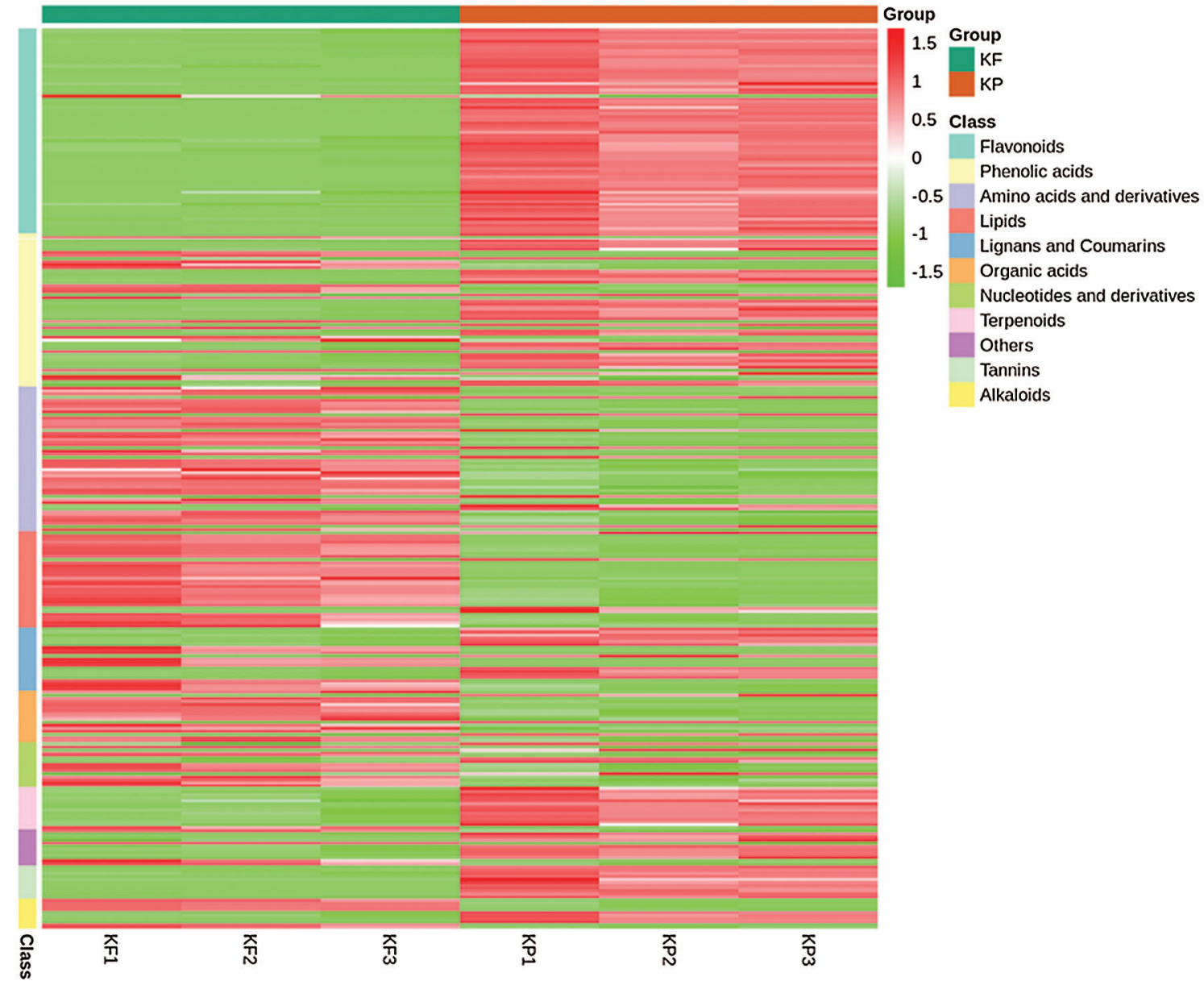

(b)
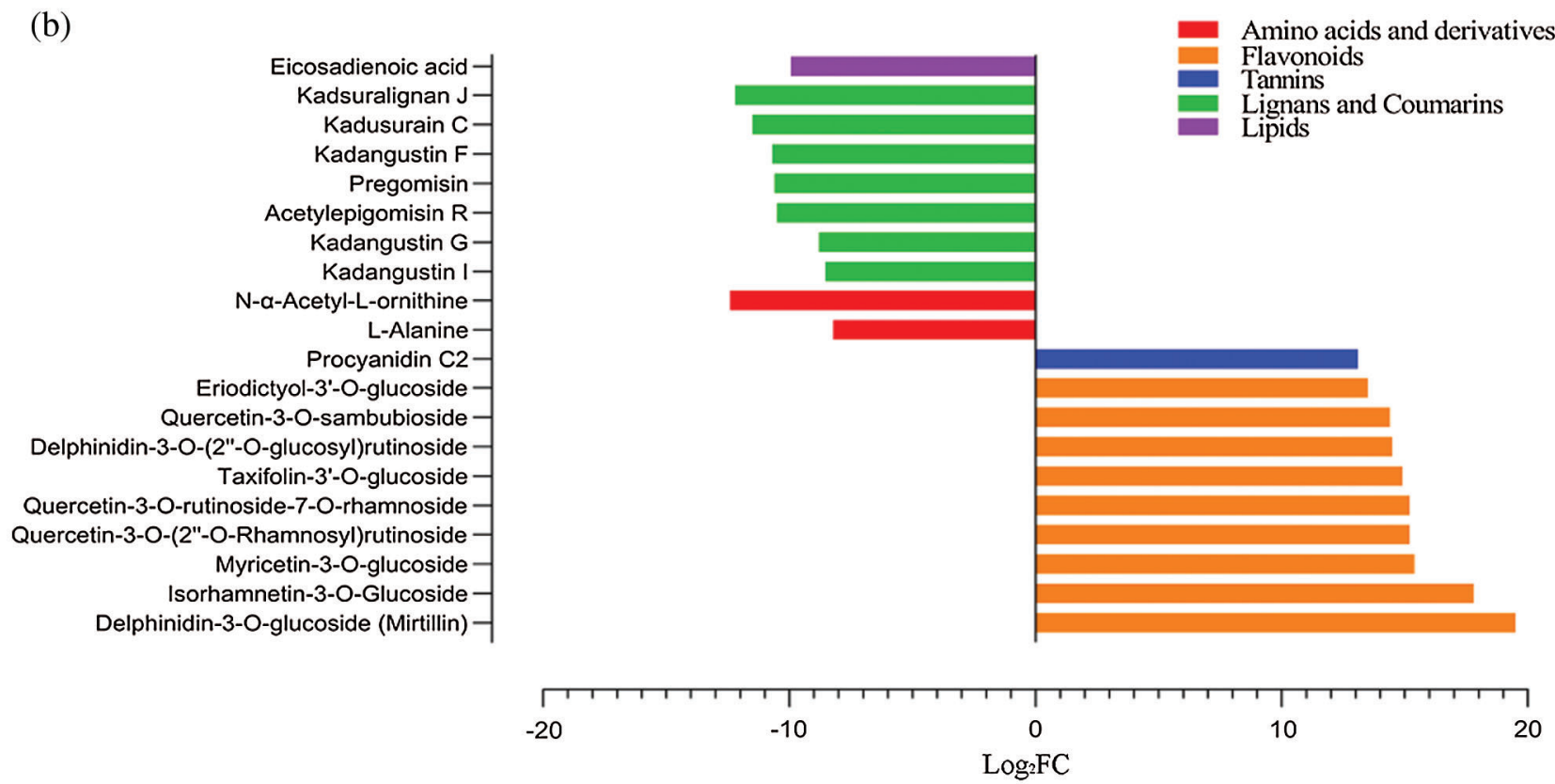

FIGURE 6. Identification of differentially accumulated metabolites (DAMs) between peel (KP) and pulp (KF), KF vs. KP.

(a) Heatmap representing accumulation profile of DAMs in peel and pulp tissues. (b) Top 20 metabolites differentially accumulated between $\mathrm{KP}$ and $\mathrm{KF}$, where the $\mathrm{x}$-axis represents $\log _{2} \mathrm{FC}$ of ion intensity.

cardiovascular protective effect (Hertog et al., 1993; Plumb et al., 2002).

In the seed, an abundance of lipids and lignans was observed. Previous reports suggested the significance of lipid and lignan compositions in the black tiger plant (Gao et al., 2008a; Li et al., 2006; Mulyaningsih et al., 2010; Shao et al., 2020). Seed and peel of black tiger fruit are generally considered biowaste; however, the abundance of bioactive 
(a)

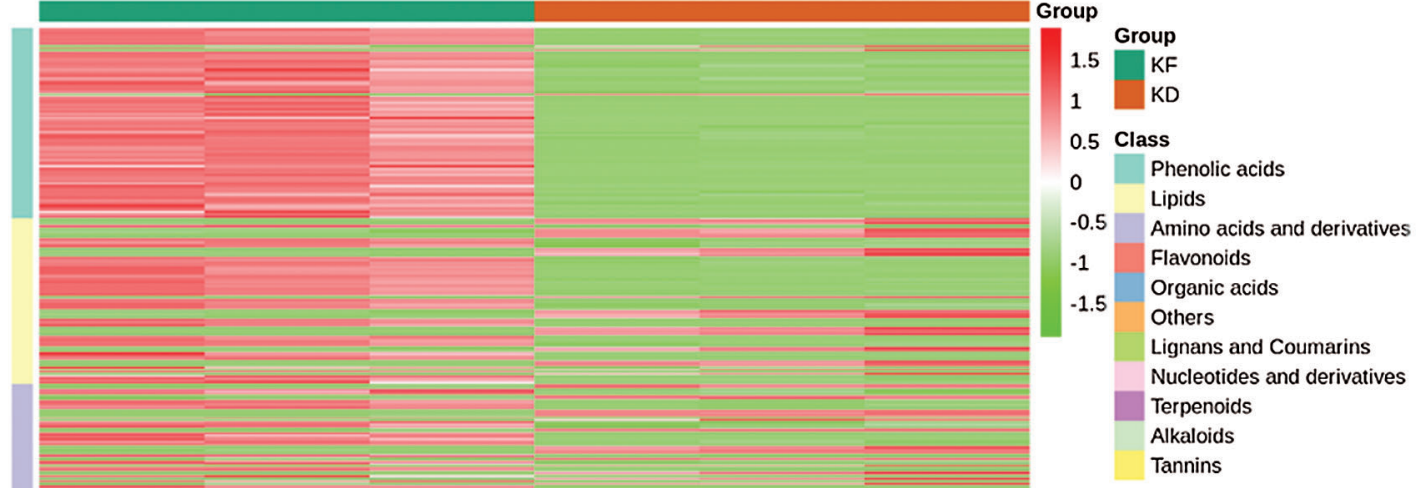

(b)

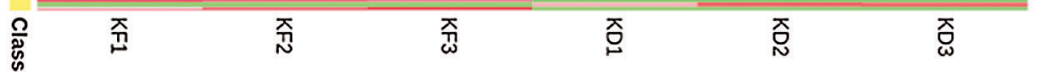

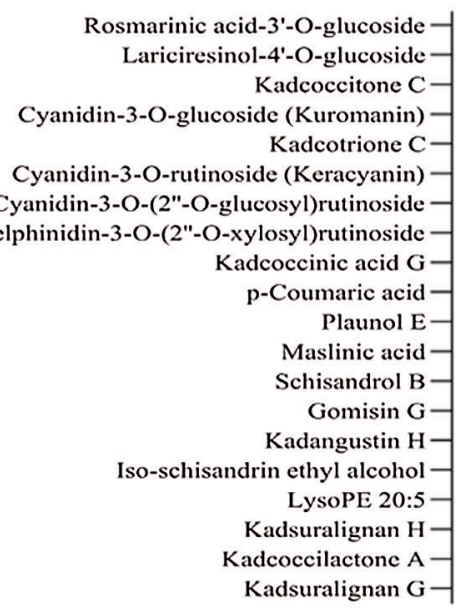

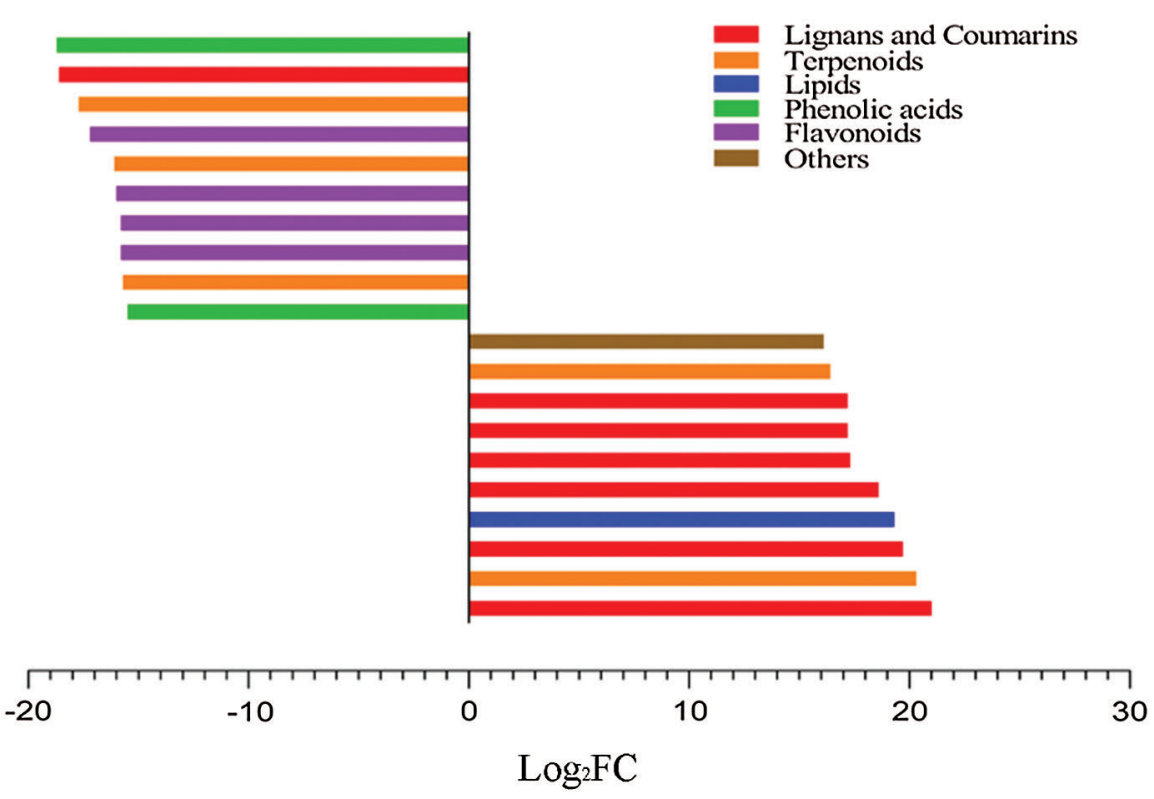

FIGURE 7. Identification of differentially accumulated metabolites (DAMs) between the seed (KD) and pulp (KF), KF vs. KD.

(a) Heatmap representing accumulation profile of the DAMs in seed and pulp tissues. (b) Top 20 metabolites differentially accumulated between $\mathrm{KD}$ and $\mathrm{KF}$, where the $\mathrm{x}$-axis represents $\log _{2} \mathrm{FC}$ of ion intensity.

compounds in both tissues, as disclosed in this study, suggests possible valorization of these fruit parts. The most prominent lignans found in the seed were kadangustin E, pregomisin, kadangustin G, kadsuralignan $\mathrm{G}$, kadangustin $\mathrm{F}$, kadangustin $\mathrm{D}$, kadusurain $\mathrm{C}$, and kadsuralignan $\mathrm{J}$.
Previously published statistics by Liu et al. (2019) on comparative authentication of Kadsura crude drug suggested identification of kadangustin $\mathrm{L}$ and kadangustin $\mathrm{E}$ in $K$. coccinea. Gao et al. (2012) emphasized lignans and triterpenoids as principal bioactive compounds present in 
(a)
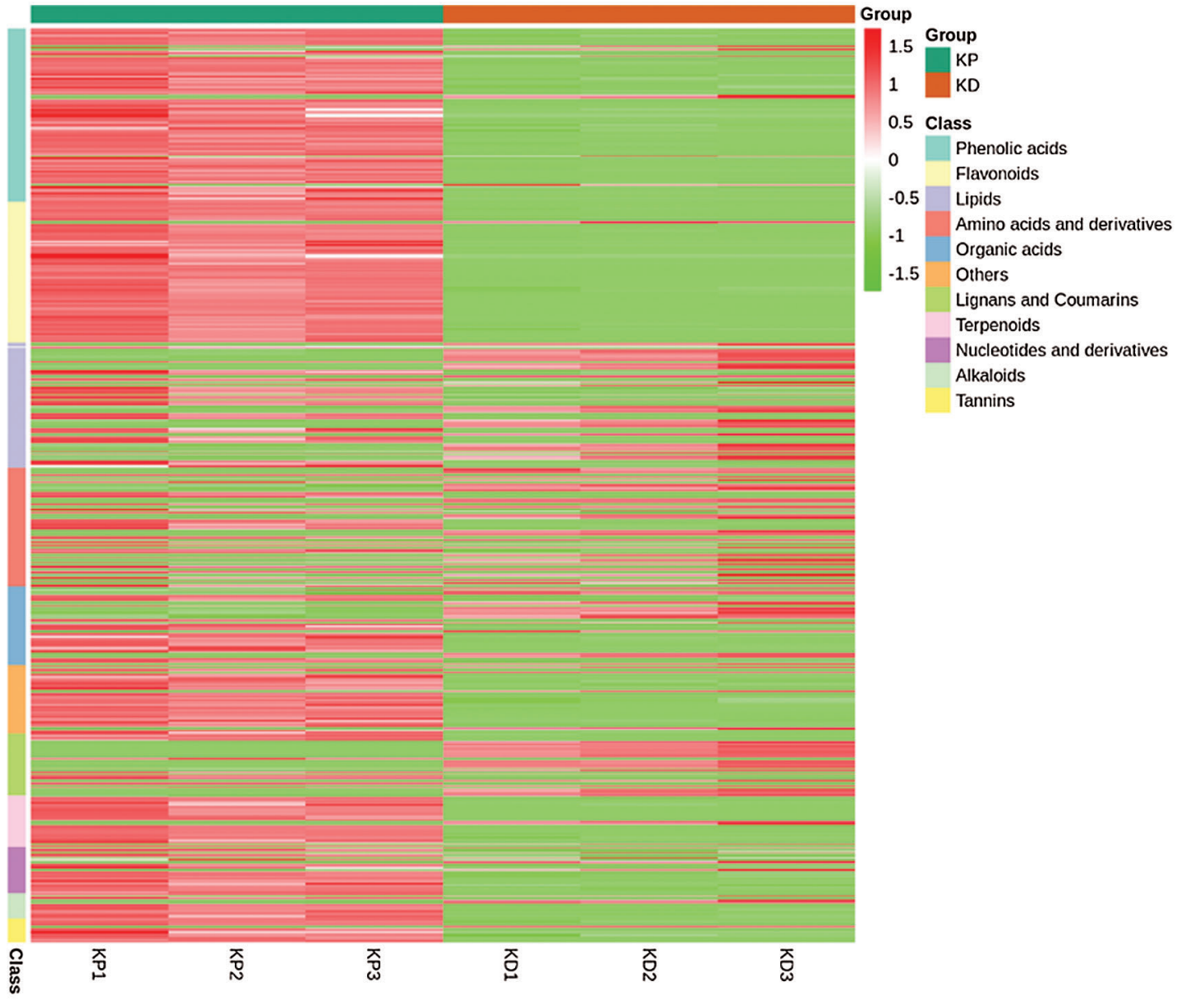

(b)

Cyanidin-3-O-glucoside (Kuromanin) Delphinidin-3-O-(2"-O-xylosyl)rutinoside Lariciresinol-4'-O-glucosideRosmarinic acid-3'-O-glucoside Quercetin-3-O-rutinoside (Rutin) Cyanidin-3-O-rutinoside (Keracyanin) Delphinidin-3-O-glucoside (Mirtillin) Cyanidin-3-O-(2"-O-glucosyl)rutinoside4-Aminobenzoic acidEpicatechin Acetylepigomisin $\mathrm{R}$ Kadsuralignan $\mathrm{H}-$ Kadangustin $\mathrm{I}$ Kadcoccilactone AKadsuralignan $\mathbf{J}$ Kadusurain $\mathrm{C}$ Kadangustin $\mathrm{F}$ Kadsuralignan $\mathrm{G}$ Kadangustin $\mathrm{G}-$ Pregomisin-

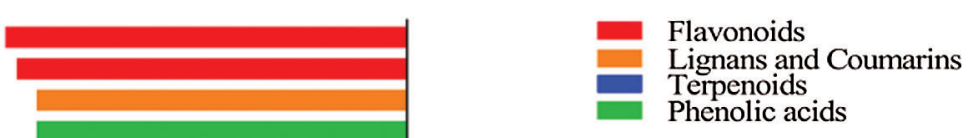

Lignans and Phenolic acids

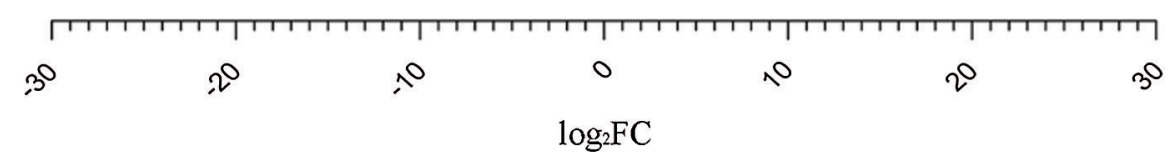

FIGURE 8. Identification of differentially accumulated metabolites (DAMs) between the seed (KD) and peel (KP), KP vs. KD.

(a) Heatmap representing the metabolite accumulation levels of the DAMs in seed and peel tissues. (b) Top 20 metabolites differentially accumulated between $\mathrm{KD}$ and $\mathrm{KP}$, where the $\mathrm{x}$-axis represents $\log _{2} \mathrm{FC}$ of ion intensity.

$K$. coccinea with significant bioactivities related to anti-HIV, immunodeficiency, cytotoxicity, and antiproliferative effects. Kadsura genus is a rich source of lignans. Previously, reports highlighted an abundance of lignans in $K$. angustifolia
(Gao et al., 2008b; Liu et al., 2019), K. philippinensis (Shen et al., 2009), K. longipedunculata and K. heteroclite (Liu et al., 2019). In contrast with a previous report (Liu et al., 2019) suggesting a low abundance of kadangustin $\mathrm{E}$ in the stem of 
K. coccinea, our results suggest a significant abundance of kadangustin E in the seed. Pregomisin (Yadav et al., 2019), kadangustin G (Gao et al., 2008b), kadsuralignan G (Goh et al., 2013), and kadsuralignan J (Liu et al., 2018b) have also been reported for their significant effects on human health and used in traditional medicine. Plant lipids are an excellent dietary source for human consumption (Ursin, 2003). Both pulp and seed tissues showed strong accumulation of linoleic acid, lysoPC 18:2, lysoPC 18:2(2n isomer), $\gamma$-linolenic acid, $\alpha$ linolenic acid, and stearic acid.

\section{Conclusions}

Our study clarified the metabolome landscapes of the fruit peel, seed, and pulp in $K$. coccinea. Comparative metabolome analysis suggests that the peel and seed tissues of $K$. coccinea are enriched in bioactive secondary metabolites, especially flavonoids and lignans, and can be further valorized as human dietary sources or raw materials in the pharmaceutical industry. Furthermore, a multi-omics approach can decipher the biosynthesis pathways for identified key metabolites to improve their bioavailability. Quantification and biological activities of the identified metabolites can be resourceful for future studies.

Availability of Data and Materials: All data generated or analyzed during this study are included in this published article (and its supplementary information files).

Author Contributions: JG and KX conceived and designed the study; JG, WZ, and WL collected the samples and performed the experiments. JG analyzed the data and drafted the manuscript. KX provided financial support, supervised the study, and revised the first drafts of the manuscript. All authors have read and approved the final version of this manuscript.

Supplementary Material: The supplementary material is available online at DOI: 10.32604/biocell.2022.016253.

Ethics Approval: Not applicable.

Funding Statement: This work was funded by the Key Project of Science and Technology Program of Guizhou Province: Poverty Alleviation Model and Technology demonstration for Ecoindustries Derivated from the karst desertification control (No. 54112017 Qiankehe Pingtai Rencai), the National Key Research and Development Program during the $13^{\text {th }}$ Five-Year Plan Period of China [No. 2016YFC0502601] and the Guizhou Province Science and Technology Plan Project "Guizhou medicinal and food dual-purpose fruit cold rice ball highquality cultivation and product development (Qian Science and Technology Cooperation Support [2016] No. 2627).

Conflicts of Interest: The authors declare that they have no conflicts of interest to report regarding the present study.

\section{References}

Bonanome A, Grundy SM (1988). Effect of dietary stearic acid on plasma cholesterol and lipoprotein levels. New England Journal of Medicine 318: 1244-1248.
Burdge G (2006). Metabolism of a-linolenic acid in humans. Prostaglandins, Leukotrienes and Essential Fatty Acids 75: 161-168.

Cao L, Shehla N, Tasneem S, Cao M, Sheng W, Jian Y, Li B, Peng C, Choudhary MI, Atta-ur-Rahman, Liao DF, Wang W (2019). New cadinane sesquiterpenes from the stems of Kadsura heteroclita. Molecules 24: 1664.

Cásedas G, Les F, López V (2020). Anthocyanins: plant pigments, food ingredients or therapeutic agents for the CNS? A mini-review focused on clinical trials. Current Pharmaceutical Design 26: 1790-1798.

Chen W, Gong L, Guo Z, Wang W, Zhang H, Liu X, Yu S, Xiong L, Luo J (2013). A novel integrated method for large-scale detection, identification, and quantification of widely targeted metabolites: application in the study of rice metabolomics. Molecular Plant 6: 1769-1780.

Deng G, Sheng O, Bi FC, Li CY, Dou TX, Dong T, Yang QS, Gao HJ, Liu J, Zhong XH (2020). Metabolic profiling in banana pseudo-stem reveals a diverse set of bioactive compounds with potential nutritional and industrial applications. Phyton 89: 1101-1130.

Dini C, Zaro MJ, Viña SZ (2019). Bioactivity and functionality of anthocyanins: a review. Current Bioactive Compounds 15: 507-523.

Dong J, Cai X, Zhao L, Xue X, Zou L, Zhang X, Liang X (2010). Lysophosphatidylcholine profiling of plasma: Discrimination of isomers and discovery of lung cancer biomarkers. Metabolomics 6: 478-488.

Dossa K, Zhou R, Li DH, Liu AL, Qin L, Marie AM, Su RQ, Zhang YJ, Wang JQ, Gao Y, Zhang XR, You J (2020). A novel motif in the 5'-UTR of an orphan gene 'Big Root Biomass' modulates root biomass in sesame. Plant Biotechnology Journal 19: 1065-1079.

El-Ishaq A, Obirinakem S (2015). Effect of temperature and storage on vitamin $\mathrm{C}$ content in fruits juice. International Journal of Chemical and Biomolecular Science 1: 17-21.

Erb M, Kliebenstein DJ (2020). Plant secondary metabolites as defenses, regulators, and primary metabolites: the blurred functional trichotomy. Plant Physiology 184: 39-52.

Fan YY, Chapkin RS (1998). Importance of dietary $\gamma$-linolenic acid in human health and nutrition. Journal of Nutrition 128: 1411-1414.

Fiehn O, Wohlgemuth G, Scholz M, Kind T, Lee DY, Lu Y, Moon S, Nikolau B (2008). Quality control for plant metabolomics: reporting MSI-compliant studies. Plant Journal 53: 691-704.

Gao XM, Pu JX, Huang SX, Lu Y, Lou LG, Li RT, Xiao WL, Chang Y, Sun HD (2008a). Kadcoccilactones A-J, triterpenoids from Kadsura coccinea. Journal of Natural Products 71: $1182-1188$

Gao XM, Pu JX, Huang SX, Yang LM, Huang H, Xiao WL, Zheng YT, Sun HD (2008b). Lignans from Kadsura angustifolia. Journal of Natural Products 71: 558-563.

Gao XM, Pu JX, Zhao Y, Yang LB, Sun HD (2012). Lignans from Kadsura angustifolia and Kadsura coccinea. Journal of Asian Natural Products Research 14: 129-134.

Goh MJ, Lee HK, Cheng L, Kong DY, Yeon JH, He QQ, Cho JC, Na YJ (2013). Depigmentation effect of kadsuralignan $f$ on melan-a murine melanocytes and human skin equivalents. International Journal of Molecular Sciences 14: 1655-1666.

Hao J, Zhu H, Liu S, Li H (2014). Characterization of anthocyanins in fruit of Kadsura coccinea (Lem.) AC Smith by UPLC/Q-TOFMS analysis and evaluation of stability of the major anthocyanins. Food Analytical Methods 7: 1312-1322. 
Hertog MG, Feskens EJ, Kromhout D, Hollman P, Katan M (1993). Dietary antioxidant flavonoids and risk of coronary heart disease: The Zutphen Elderly Study. Lancet 342: 1007-1011.

Hu ZX, Shi YM, Wang WG, Tang JW, Zhou M, Du X, Zhang YH, $\mathrm{Pu}$ JX, Sun HD (2016). Structural characterization of kadcoccinin A: A sesquiterpenoid with a tricyclo [4.4. 0. 03, 10] decane scaffold from Kadsura coccinea. Organic Letters 18: 2284-2287.

Ikeda I, Kobayashi M, Hamada T, Tsuda K, Goto H, Imaizumi K, Nozawa A, Sugimoto A, Kakuda T (2003). Heat-epimerized tea catechins rich in gallocatechin gallate and catechin gallate are more effective to inhibit cholesterol absorption than tea catechins rich in epigallocatechin gallate and epicatechin gallate. Journal of Agricultural and Food Chemistry 51: 7303-7307.

Kuo YH, Wu MD, Huang RL, Kuo LMY, Hsu YW, Liaw CC, Hung CC, Shen YC, Ong CW (2005). Antihepatitis activity (antiHBsAg and anti-HBeAg) of C19 homolignans and six novel C18 dibenzocyclooctadiene lignans from Kadsura japonica. Planta Medica 71: 646-653.

Li HR, Feng YL, Yang ZG, Wang J, Daikonya A, Kitanaka S, Xu LZ, Yang SL (2006). New lignans from Kadsura coccinea and their nitric oxide inhibitory activities. Chemical and Pharmaceutical Bulletin 54: 1022-1025.

Li H, Wang L, Yang Z, Kitanaka S (2007). Kadsuralignans H-K from Kadsura coccinea and their nitric oxide production inhibitory effects. Journal of Natural Products 70: 1999-2002.

Liu J, Pandey P, Wang X, Qi X, Chen J, Sun H, Zhang P, Ding Y, Ferreira D, Doerksen RJ (2018a). Hepatoprotective dibenzocyclooctadiene and tetrahydrobenzocyclooctabenzofuranone lignans from Kadsura longipedunculata. Journal of Natural Products 81: 846-857.

Liu J, Qi Y, Lai H, Zhang J, Jia X, Liu H, Zhang B, Xiao P (2014). Genus Kadsura, a good source with considerable characteristic chemical constituents and potential bioactivities. Phytomedicine 21: 1092-1097.

Liu J, Wei X, Zhang X, Qi Y, Zhang B, Liu H, Xiao P (2019). A comprehensive comparative study for the authentication of the Kadsura crude drug. Frontiers in Pharmacology 9: 1576.

Liu Y, Yang Y, Tasneem S, Hussain N, Daniyal M, Yuan H, Xie Q, Liu B, Sun J, Jian Y (2018b). Lignans from Tujia ethnomedicine Heilaohu: chemical characterization and evaluation of their cytotoxicity and antioxidant activities. Molecules 23: 2147.

Matsumoto H, Inaba H, Kishi M, Tominaga S, Hirayama M, Tsuda T (2001). Orally administered delphinidin 3-rutinoside and cyanidin 3-rutinoside are directly absorbed in rats and humans and appear in the blood as the intact forms. Journal of Agricultural and Food Chemistry 49: 1546-1551.

Matsuo T, Hanamure N, Shimoi K, Nakamura Y, Tomita I (1994). Identification of (+)-gallocatechin as a bio-antimutagenic compound in Psidium guava leaves. Phytochemistry 36: 1027-1029.

Mulyaningsih S, Youns M, El-Readi MZ, Ashour ML, Nibret E, Sporer F, Herrmann F, Reichling J, Wink M (2010). Biological activity of the essential oil of Kadsura longipedunculata (Schisandraceae) and its major components. Journal of Pharmacy and Pharmacology 62: 1037-1044.

Nasser H, Kader HM, Al Mughairbi F (2019). Neuroprotective effects of kuromanin chloride (anthocyanin) against glutamatergic excitotoxicity in neuroblastoma cells. Frontiers in Human Neuroscience 13. DOI 10.3389/conf.fnhum.2019.3229.00035. https://www.frontiersin.org/10.3389\%2fconf.fnhum.2019. 229.00035/event_abstract.

Olivas-Aguirre FJ, Rodrigo-García J, Martínez-Ruiz NdR, Cárdenas-Robles AI, Mendoza-Díaz SO, Alvarez-Parrilla E, González-Aguilar GA, De la Rosa LA, Ramos-Jiménez A, Wall-Medrano A (2016). Cyanidin-3-O-glucoside: Physicalchemistry, foodomics and health effects. Molecules 21: 1264.

Olthof MR, Hollman PC, Buijsman MN, Van Amelsvoort JM, Katan MB (2003). Chlorogenic acid, quercetin-3-rutinoside and black tea phenols are extensively metabolized in humans. Journal of Nutrition 133: 1806-1814.

Owis AI, El-Hawary MS, El Amir D, Aly OM, Abdelmohsen UR, Kamel MS (2020). Molecular docking reveals the potential of Salvadora persica flavonoids to inhibit COVID-19 virus main protease. RSC Advances 10: 19570-19575.

Plumb G, De Pascual-Teresa S, Santos-Buelga C, Rivas-Gonzalo JC, Williamson G (2002). Antioxidant properties of gallocatechin and prodelphinidins from pomegranate peel. Redox Report 7: 41-46.

Ren C, Liu J, Zhou J, Liang H, Wang Y, Sun Y, Ma B, Yin Y (2018). Lipidomic analysis of serum samples from migraine patients. Lipids in Health and Disease 17: 22.

Sawada Y, Akiyama K, Sakata A, Kuwahara A, Otsuki H, Sakurai T, Saito K, Hirai MY (2009). Widely targeted metabolomics based on large-scale MS/MS data for elucidating metabolite accumulation patterns in plants. Plant and Cell Physiology 50: 37-47.

Shao SY, Qi XZ, Sun H, Li S (2020). Hepatoprotective lignans and triterpenoids from the roots of Kadsura longipedunculata. Fitoterapia 142: 104487.

Shen YC, Lin YC, Cheng YB, Chiang MY, Liou SS, Khalil AT (2009). Dibenzocyclooctadiene lignans from Kadsura philippinensis. Phytochemistry 70: 114-120.

Sritalahareuthai V, Aursalung A, On-Nom N, Temviriyanukul P, Charoenkiatkul S, Suttisansanee U (2020a). Nutritional composition of conserved Kadsura spp. plants in Northern Thailand. Heliyon 6: e04451.

Sritalahareuthai V, Temviriyanukul P, On-Nom N, Charoenkiatkul S, Suttisansanee U (2020b). Phenolic profiles, antioxidant, and inhibitory activities of Kadsura heteroclita (Roxb.) Craib and Kadsura coccinea (Lem.) A.C. Sm. Foods 9: 1222.

Sun C, Zheng Y, Chen Q, Tang X, Jiang M, Zhang J, Li X, Chen K (2012). Purification and anti-tumour activity of cyanidin-3O-glucoside from Chinese bayberry fruit. Food Chemistry 131: $1287-1294$.

Sun J, Jiang Y, Amin I, Li Z, Prasad K, Duan X, Yang B, Xu L (2011). An exotic fruit with high nutritional value: Kadsura coccinea fruit. International Food Research Journal 18: 651-657.

Sun J, Yao J, Huang S, Long X, Wang J, García-García E (2009). Antioxidant activity of polyphenol and anthocyanin extracts from fruits of Kadsura coccinea (Lem.) A.C. Smith. Food Chemistry 117: 276-281.

Ursin VM (2003). Modification of plant lipids for human health: development of functional land-based omega-3 fatty acids. Journal of Nutrition 133: 4271-4274.

Wang J, Guo D, Han D, Pan X, Li J (2020a). A comprehensive insight into the metabolic landscape of fruit pulp, peel, and seed in two longan (Dimocarpus longan Lour.) varieties. International Journal of Food Properties 23: 1527-1539.

Wang M, Jiang S, Yuan H, Zafar S, Hussain N, Jian Y, Li B, Gong L, Peng C, Liu C (2020b). A review of the phytochemistry and pharmacology of Kadsura heteroclita, an important plant in 
Tujia ethnomedicine. Journal of Ethnopharmacology 268: 113567.

Woo MH, Nguyen DH, Choi JS, Park SE, Thuong PT, Min BS, Le DD (2020). Chemical constituents from the roots of Kadsura coccinea with their protein tyrosine phosphatase $1 \mathrm{~B}$ and acetylcholinesterase inhibitory activities. Archives of Pharmacal Research 43: 204-213.

Xu L, Xu Z, Wang X, Wang B, Liao X (2020). The application of pseudotargeted metabolomics method for fruit juices discrimination. Food Chemistry 316: 126278.

Xu W, Luo G, Yu F, Jia Q, Zheng Y, Bi X, Lei J (2018). Characterization of anthocyanins in the hybrid progenies derived from Iris dichotoma and I. domestica by HPLCDAD-ESI/MS analysis. Phytochemistry 150: 60-74.
Yadav M, Mohanta R, Yadav K (2019). Ethno pharmacological approach of anti-obesity medicinal plants: A review. Journal of Pharmacognosy and Phytochemistry 8: 1793-1798.

Yang X, Xia X, Zhang Z, Nong B, Zeng Y, Wu Y, Xiong F, Zhang Y, Liang H, Pan Y, Dai (2019a). Identification of anthocyanin biosynthesis genes in rice pericarp using PCAMP. Plant Biotechnology Journal 17: 1700-1702.

Yang YP, Hussain N, Zhang L, Jia YZ, Jian YQ, Li B, Choudhary MI, Rahman AU, Wang W (2020). Kadsura coccinea: A rich source of structurally diverse and biologically important compounds. Chinese Herbal Medicines 12: 214-223.

Yang Y, Liu Y, Daniyal M, Yu H, Xie Q, Li B, Jian Y, Man R, Wang S, Zhou X (2019b). New lignans from roots of Kadsura coccinea. Fitoterapia 139: 104368.

\section{Supplementary Materials}
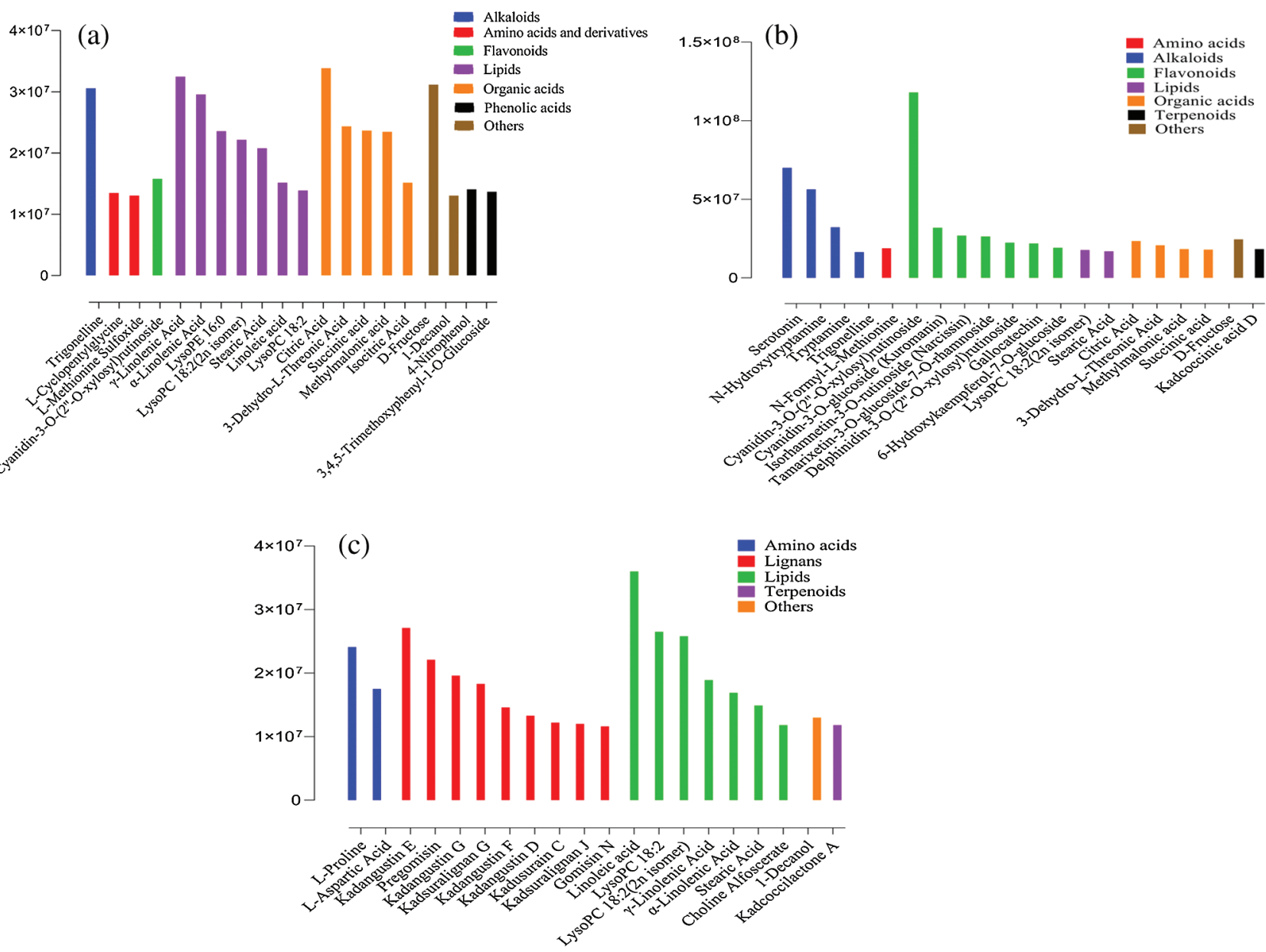

FIGURE S1. Top 20 metabolites and their ion intensity in respective tissues.

(a) Top 20 metabolites and their corresponding classification in pulp (KF), (b) Top 20 metabolites and their corresponding classification in peel (KP), (c) Top 20 metabolites and their corresponding classification in seed (KD) of the black tiger. 


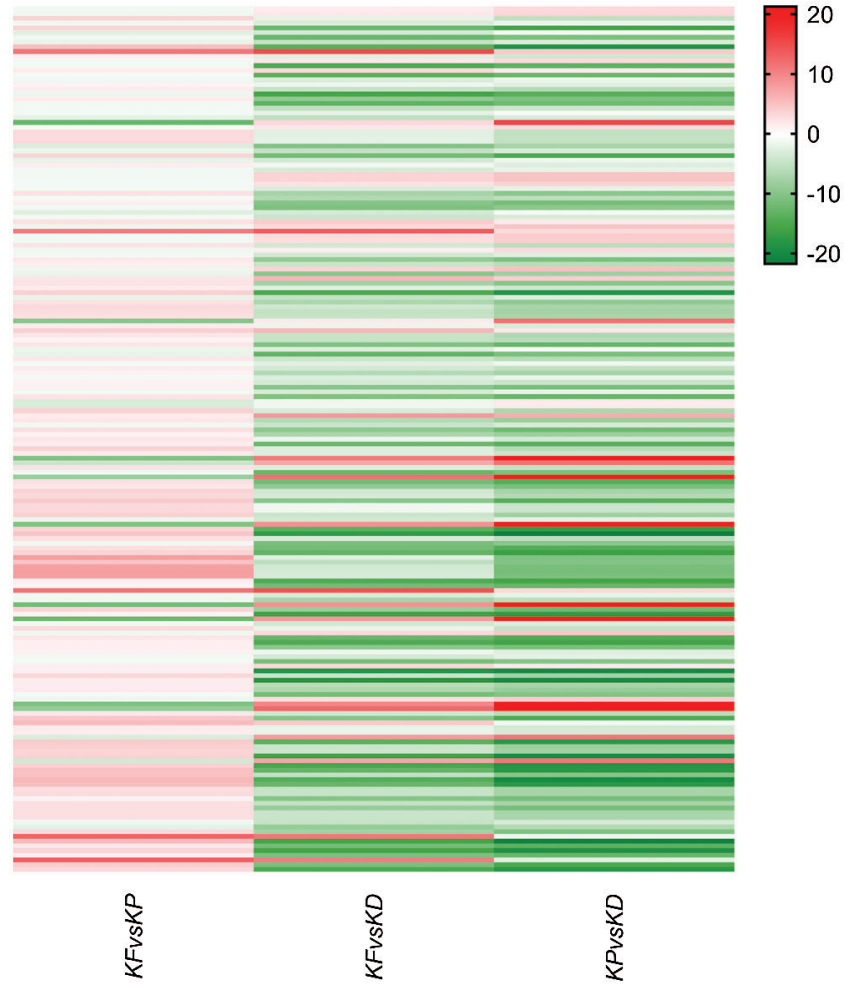

FIGURE S2. Heatmap representing the differential accumulation of metabolites in three group comparisons $\mathrm{KF} v s$. KP, KF vs. KD, and $\mathrm{KP}$ vs. $\mathrm{KD}$, where $\mathrm{KF}, \mathrm{KP}$, and $\mathrm{KD}$ correspond to black tiger fruit pulp, peel, and seed tissues, respectively.

\section{TABLE S1}

Metabolic landscape of black tiger fruit and respective tissues, i.e., peel, pulp, and seed

\section{TABLE S2}

Differentially expressed metabolites between pulp and peel tissues (KF vs. KP) of black tiger fruit.

\section{TABLE S3}

Differentially expressed metabolites between pulp and seed tissues (KF vs. KD) of black tiger fruit.

\section{TABLE S4}

Differentially expressed metabolites between peel and seed tissues (KP vs. KD) of black tiger fruit.

\section{TABLE S5}

Conserved differentially expressed metabolites between different tissues of black tiger fruit. 